\title{
Plasmodium vivax metacaspase 1 (PvMCA1) catalytic domain is conserved in field isolates from Brazilian Amazon
}

\author{
Hugo Amorim dos Santos de Souza', Victor Fernandes Escafa', Carolina Moreira Blanco', \\ Bárbara de Oliveira Baptista', Jenifer Peixoto de Barros', Evelyn Ketty Pratt Riccio', \\ Aline Beatriz Mello Rodrigues ${ }^{2}$, Gisely Cardoso de Melo ${ }^{3,4}$, \\ Marcus Vinícius Guimarães de Lacerda ${ }^{4,5}$, Rodrigo Medeiros de Souza ${ }^{6}$, \\ Josué da Costa Lima-Junior ${ }^{7}$, Ana Carolina Ramos Guimarães ${ }^{2}$, Fabio Faria da Mota ${ }^{8}$, \\ João Hermínio Martins da Silva9 ${ }^{9}$ Cláudio Tadeu Daniel-Ribeiro', Lilian Rose Pratt-Riccio, \\ Paulo Renato Rivas Totino ${ }^{1 /+}$
}

${ }^{1}$ Fundação Oswaldo Cruz-Fiocruz, Instituto Oswaldo Cruz, Laboratório de Pesquisa em Malária, Rio de Janeiro, RJ, Brasil ${ }^{2}$ Fundação Oswaldo Cruz-Fiocruz, Instituto Oswaldo Cruz, Laboratório de Genômica Funcional e Bioinformática, Rio de Janeiro, RJ, Brasil ${ }^{3}$ Universidade do Estado do Amazonas, Manaus, AM, Brasil

${ }^{4}$ Fundação de Medicina Tropical Heitor Vieira Dourado, Instituto de Pesquisa Clínica Carlos Borborema, Manaus, AM, Brasil ${ }^{5}$ Fundação Oswaldo Cruz-Fiocruz, Instituto Leônidas and Maria Deane, Manaus, AM, Brasil

${ }^{6}$ Universidade Federal do Acre, Centro de Pesquisa em Doenças Infecciosas, Centro Multidisciplinar, Rio Branco, AC, Brasil

7Fundação Oswaldo Cruz-Fiocruz, Instituto Oswaldo Cruz, Laboratório de Imunoparasitologia, Rio de Janeiro, RJ, Brasil

${ }^{8}$ Fundação Oswaldo Cruz-Fiocruz, Instituto Oswaldo Cruz, Laboratório de Biologia Computacional e Sistemas, Rio de Janeiro, RJ, Brasil

${ }^{9}$ Fundação Oswaldo Cruz-Fiocruz, Eusébio, CE, Brasil

In the present study, we investigated the genetic diversity of Plasmodium vivax metacaspase 1 ( $P v$ MCA1) catalytic domain in two municipalities of the main malaria hotspot in Brazil, i.e., the Juruá Valley, and observed complete sequence identity among all $P$. vivax field isolates and the Sal-1 reference strain. Analysis of $P v \mathrm{MCA} 1$ catalytic domain in different $P$. vivax genomic sequences publicly available also revealed a high degree of conservation worldwide, with very few amino acid substitutions that were not related to putative histidine and cysteine catalytic residues, whose involvement with the active site of protease was herein predicted by molecular modeling. The genetic conservation presented by $P v \mathrm{MCA} 1$ may contribute to its eligibility as a druggable target candidate in vivax malaria.

Key words: P. vivax - metacaspase - genetic diversity

The emergence and spread of drug-resistant parasites constitute major obstacles to malaria control in the world, ${ }^{(1)}$ where Plasmodium falciparum and $P$. vivax are the most important species causing malaria. ${ }^{(2)}$ Throughout the history of antimalarial therapy, $P$. falciparum has been notorious for its capacity to develop resistance to diverse antimalarial drugs, including chloroquine, sulfadoxine/pyrimethamine and, more recently, artemisinin in Southeast Asia. ${ }^{(1,3)}$ Nevertheless, emergence of drugresistant $P$. vivax has also been documented since the late 1980s in Papua New Guinea and, ${ }^{(4,5)}$ currently, therapeutic failure of chloroquine, which combined with primaquine comprise the first-line treatment for vivax malaria, is reported in many vivax-endemic countries, ${ }^{(6,7)}$ while the magnitude of primaquine tolerance remains largely unknown. ${ }^{(8)}$ These facts highlight the urgent need

doi: 10.1590/0074-02760200584

Financial support: IOC (FIOCRUZ), FAPERJ, CAPES.

MVGL and CTDR receive a Productivity Research Fellowship from the

CNPq, Brazil; CTDR is a "Cientista do Nosso Estado" from FAPERJ.

+ Corresponding author: prtotino@ioc.fiocruz.br

(D) https://orcid.org/0000-0002-4790-3317

Received 13 November 2020

Accepted 04 May 2021 for the development of novel compounds targeting plasmodial molecular pathways with mechanisms of action different from the classical antimalarial drugs.

In this scenario, proteases playing significant roles in parasite survival have been for long time considered molecular targets for antimalarial drug development and, ${ }^{(9,10)}$ more recently, members of metacaspase family, whose activity was already suggested to be implicated in both growth and cell death of Plasmodium and other protozoan parasites, have emerged as novel candidates. $(11,12)$ Metacaspases are cysteine proteases belonging to the $\mathrm{C} 14$ family that are found in genome of protists, fungi, algae and plants. They present structural similarity to metazoan caspases, both having a conserved His-Cys catalytic dyad in the large (p20) domain, but different substrate specificity. ${ }^{(13)}$ In Plasmodium genomes, three metacaspases (MCA1-3) occur ${ }^{(14)}$ and a study performed with field isolates from Mauritania, Sudan and Oman showed that $P$. vivax metacaspase 1 ( $P v$ MCA1) can present single nucleotide polymorphisms in the putative HisCys catalytic residues, ${ }^{(15)}$ which in some extent can be a factor limiting the eligibility of $P v \mathrm{MCA} 1$ as a novel drug target for vivax malaria. In the present study, therefore, we investigated the genetic diversity of the $P v \mathrm{MCA} 1$ catalytic domain in a $P$. vivax population from the main malaria hotspot in Brazil - a country where this plasmodial species is highly prevalent $(\sim 90 \%)$. $^{(2,16)}$ 
Study area and sample collection - P. vivax parasite isolates were collected from two municipalities [Cruzeiro do Sul (CZS; $\left.7^{\circ} 37^{\prime} 51^{\prime \prime} \mathrm{S}, 72^{\circ} 40^{\prime} 12^{\prime \prime} \mathrm{W}\right)$ and Mâncio Lima (ML; 0 $\left.7^{\circ} 36^{\prime} 50^{\prime \prime} \mathrm{S}, 72^{\circ} 53^{\prime} 4^{\prime \prime} \mathrm{W}\right)$ ] situated in the Juruá Valley, Northwest of the Acre State, Brazilian Amazon, from June to August 2016 and 2018. Covering together a surface area of 12,597 km² (CZS: 7,925 km²; ML: 4,672 $\mathrm{km}^{2}$ ), these municipalities are among the endemic areas with the highest annual parasite incidence (API) (positive blood slides per 1,000 inhabitants) in Brazil, presenting an epidemiological profile with sustained high transmission over the last decade. For reference, the Brazilian Ministry of Health considers high risk areas those with API $\geq 50$, and Mâncio Lima and Cruzeiro do Sul registered APIs of 436.4 and 231.9 in 2016 and 422.8 and 147.5 in 2018, respectively. In these areas, eightythree $P$. vivax mono-infected individuals living in different localities and presenting uncomplicated malaria were diagnosed through microscopic examination of Giemsa-stained thick blood smears and, then, a single blood sample was collected in EDTA tubes from each individual before initiation of treatment. Samples were centrifuged at $350 \mathrm{~g}$ for $10 \mathrm{~min}$ to remove plasma and cell pellet was preserved at $-20^{\circ} \mathrm{C}$ in glycerolyte solution (1:2). Written informed consent was obtained from all donors and the study was reviewed and approved by the Oswaldo Cruz Foundation Ethical Committee and the National Ethical Committee of Brazil (CEP-FIOCRUZ CAAE 46084015.1.0000.5248). All $P$. vivax infections were later confirmed by polymerase chain reaction (PCR) assay, as previously described. ${ }^{(17)}$

$D N A$ extraction and PCR amplification - parasite DNA was extracted from cryopreserved blood samples by QIAamp DNA blood midi kit (QIAgen), following the manufacturer's instructions, and stored at $-20^{\circ} \mathrm{C}$ until use. Gene segment coding for the catalytic domain of $P v \mathrm{MCA} 1$ was amplified by standard PCR method using a pair of specific primers (forward, 5'-CATGGAAACAAAAAAAAGG-3'; reverse, 5'-CGAAAACTCCATATCTTTGC-3'), as previously described. ${ }^{(15)}$ PCR was performed in a Veriti 96well Thermal Cycler (Applied Biosystems) with a total volume of $25 \mu \mathrm{L}$ reaction mixture containing $3 \mu \mathrm{L}$ genomic DNA, $10 \mathrm{pmol} / \mu \mathrm{L}$ each primer, 2.5 unit Ampli$\mathrm{Taq}^{\mathrm{TM}}$ Gold DNA polymerase, $3 \mathrm{mM} \mathrm{MgCl}$ and $2 \mu \mathrm{L}$ of 10X PCR buffer. The following conditions were used: 35 cycles at $95^{\circ} \mathrm{C}$ for $30 \mathrm{~s}, 56^{\circ} \mathrm{C}$ for $30 \mathrm{~s}$ and $72^{\circ} \mathrm{C}$ for 2 min. Amplified products were size-fractionated by electrophoresis within $2 \%$ agarose gel (Sigma) containing 0.5 $\mu \mathrm{g} / \mathrm{mL}$ of ethidium bromide and, then, were visualised by ultraviolet illumination.

Sequencing and polymorphism analysis - PCR products were purified by Wizard ${ }^{\circledR}$ SV Gel and PCR CleanUp System (Promega) following the manufacturer's instructions and sequenced in both directions using above-mentioned primers. The sequencing reaction was performed in duplicate according BigDye ${ }^{\mathrm{TM}}$ Terminator v3.1 Cycle Sequencing Kit Applied Biosystems ${ }^{\mathrm{TM}}$ using 75-100 ng of the purified PCR products and, then, the obtained products were read on a $3730 \mathrm{xl}$ DNA Analyser (Applied Biosystems). Forward and reverse sequences were compared and checked for quality by using SeqMan v.7.0.0 of the DNASTAR software package (Lasergen, Madison, WI, USA), with default parameters, following manual inspection of chromatograms to eliminate ambiguous bases. A minimum quality score of 20 (base call accuracy $\geq 99 \%$ ) was considered. Alignment of edited sequences was performed in MEGA 7 using Clustal X2 algorithm to identify polymorphisms relative to $P v \mathrm{M}$ CA1 sequence from El Salvador reference strain (Sal-1; PlasmoDB: PVX 114725). Additionally, for worldwide analysis, 112 nucleotide sequences for the complete catalytic domain of $P v \mathrm{MCA} 1$, as predicted using Pfam database (https://pfam.xfam.org/), were recovered from PlasmoDB and GenBank databases by BLAST and aligned to search for genetic variability. Metadata obtained were used to identify geographical distribution of the isolates recovered from these databases.

Molecular modeling - The sequence of G0ZIA8 (Sal1; UniProtKB) was submitted to Blast, in the search of homologous proteins. The template (4AFR, Trypanosoma brucei metacaspase) found in PDB share 38.4\% identity with G0ZIA8. After alignment with blast, the coverage is $32 \%$. The e-value was $7 \mathrm{e}-35$. The structural model of G0ZIA8 was constructed with MODELLER (https://salilab.org/modeller/). Fifty models were constructed and the best one was selected according to the DOPE score. The electrostatic surface potential was calculated by APBS software. The structural analysis was performed with Pymol.

To investigate the genetic diversity of $P v \mathrm{MCA} 1$, eighty-three wild isolates of $P$. vivax from Juruá Valley in the Brazilian Amazon were submitted to DNA sequencing for gene segment coding the consensus His372-Cys428 catalytic dyad (13). In contrast to work by Sow et al., ${ }^{(15)}$ in which the majority of isolates $(24 / 28,85.7 \%)$ presented amino acid substitution in both catalytic dyad and an upstream cysteine residue (Cys305) also inserted in the peptidase C14 domain, all the isolates herein studied showed complete nucleotide sequence identity each other as well as compared to $P$. vivax Sal-1 reference, with conservation of all the amino acid residues successfully sequenced (A288 to K446) (Fig. 1), supporting that the proteolytic activity of $P v \mathrm{MCA} 1$ may be critical to the parasite.

Since functional disruption of the single metacaspase (MCA1) in Saccharomyces cerevisiae by mutagenesis was already shown to induce in vitro drug-resistance as a result of refractoriness to apoptosis in the yeasts ${ }^{(18)}$ one possibility is that polymorphism of $P v \mathrm{MCA} 1$ could be related to chemoresistance phenomena reported in vivax malaria, whose occurrence is reported to be infrequent in Juruá Valley ${ }^{(19)}$ and that was not characterised in our cross-sectional study. Indeed, metacaspases have been implicated in apoptosis-like cell-death pathways in different microorganisms ${ }^{(2)}$ and drug-resistant strains of $P$. falciparum seem to be insensitive to choroquine-induced apoptosis $^{(21)}$ as well as to anti-cell death effect promoted by inhibition of metacaspase activity. ${ }^{(22)}$ Nevertheless, our preliminary study with recurrent infection in five $P$. vivax patients receiving appropriate antimalarial therapy (chloroquine/primaquine) at a Reference Center for ma- 


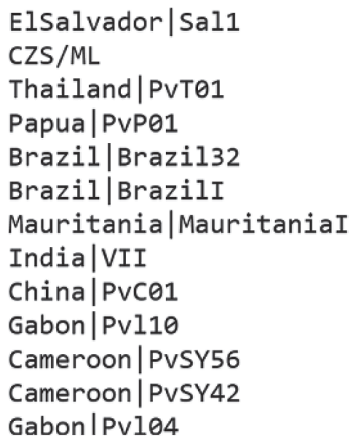

286

KKALLIGINYYGSREELSGCTNDTLRMMNLLISKYNFHDSPTSMVRLIDNESNPNYRPTR - -ALLIGINYYGSREELSGCTNDTLRMMNLLISKYNFHDSPTSMVRLIDNESNPNYRPTR KKALLIGINYYGSREELSGCTNDTLRMMNLLISKYNFHDSPTSMVRLIDNESNPNYRPTR KKALLIGINYYGSREELSGCTNDTLRMMNLLISKYNFHDSPTSMVRLIDNESNPNYRPTR KKALLIGINYYGSREELSGCTNDTLRMMNLLISKYNFHDSPTSMVRLIDNESNPNYRPTR KKALLIGINYYGSREELSGCTNDTLRMMNLLISKYNFHDSPTSMVRLIDNESNPNYRPTR KKALLIGINYYGSREELSGCTNDTLRMMNLLISKYNFHDSPTSMVRLIDNESNPNYRPTR KKALLIGINYYGSREELSGCTNDTLRMMNLLISKYNFHDSPTSMVRLIDNESNPNYRPTR KKALLIGINYYGSREELSGCTNDTLRMMNLLISKYNFHDSPTSMVRLIDNESNPNYRPTR KKALLIGINYYGSREELSGCTNDTMRMMNLLISKYNFHDSPTSMVRLIDNESNPNYRPTR KKALLIGINYYGSREELSGCTNDTMRMMNLLISKYNFHDSPTSMVRLIDNESNPNYRPTR KKALLIGINYYGSREELSGCTNDTURMMNLLISKYNFHDSPTSMVRLIDNESNPNYRPTR KKALLIGINYYGSREELSGCTNDTURMMNLLISKYNFHDSPTSMVRLIDNESNPNYRPTR

346 |KNILSALNWLTKDNQPGDVFFFLYSGHGSQQKDYTYLEDDGYNETILPCDHKTEGQIIDD | KNILSALNWLTKDNQPGDVFFFLYSGHGSQQKDYTYLEDDGYNETILPCDHKTEGQIIDD KNILSALNWLTKDNQPGDVFFFLYSGHGSQQKDYTYLEDDGYNETILPCDHKTEGQIIDD KNILSALNWLTKDNQPGDVFFFLYSGHGSQQKDYTYLEDDGYNET ILPCDHKTEGQIIDD KNILSALNWLTKDNQPGDVFFFLYSGHGSQQKDYTYLEDDGYNETILPCDHKTEGQIIDD KNILSALNWLTKDNQPGDVFFFLYSGHGSQQKDYTYLEDDGYNET ILPCDHKTEGQIIDD KNILSALNWLTKDNQPGDVFFFLYSGHGSQQKDYTYLEDDGYNETILPCDHKTEGQIIDD KNILSALNWLTKDNQPGDVFFFLYSGHGSQQKDYTYLEDDGYNETILPCDHKTEGQIIDD KNILSALNWLTKDNQPGDVFFFLYSGHGSQQKDYTYLEDDGYNET ILPCDHKTEGQIIDD KNILSALNWLTKDNQPGDVFFFLYSGHGSQQKDYTYLEDDGYNETILPCDHKTEGQIIDD KNILSALNWLTKDNEPGDVFFFLYSGHGSQQKDYTYLEDDGYNETILPCDHKTEGQIIDD KNILSALNWLTKDNEPGDVFFFLYSGHGSQQKDYTYLEDDGYNETILPCDHKTEGQIIDD KNILSALNWLTKDNEPGDVFFFLYSGHGSQQKDYTYLEDDGYNETILPCDHKTEGQIIDD

406 $406 \quad \downarrow * \quad \# \quad \# \quad 465$

ELHRFLVQPLNDGVKLIAVMDCCNAGSCIDLAYKYKLKSKKWKEVKNPFHVVCDVSQFSG ELHRFLVQPLNDGVKLIAVMDCCNAGSCIDLAYKYKLKSKK - ELHRFLVQPLNDGVKLIAVMDCCNAGSCIDLAYKYKLKSKKWKEVKNPFHVVCDVSQFSG ELHRFLVQPLNDGVKLIAVMDCCNAGSCIDLAYKYKLKSKKWKEVKNPFHVVCDVSQFSG ELHRFLVQPLNDGVKLIAVMDCCNAGSCIDLAYKYKLKSKKWKEVKNPFHVVCDVSQFSG ELHRFLVQPLNDGVKLIAVMDCCNAGSCIDLAYKYKLKSKKWKEVKNPFHVVCDVSQFSG ELHRFLVQPLNDGVKLIAVMDCCNAGSCIDLAYKYKLKSKKWKEVKNPFHVVCDVSQFSG ELHRFLVQPLNDGVKLIAVMDCCNAGSCIDLAYKYKLKSKKWKEVKNPFHVVCDVSQFSG ELHRFLVQPLNDGVKLIAVMDCCNAGSCIDLAYKYKLKSKKWKEVKNPFHVVCDVSQFSG ELHRFLVQPLNDGVKLIAVMDCCNAGSC IDLAYKYKLKSKKWKEVKNPFHVVCDVSQFSG ELHRFLVQPLNDGVKLIAVMDCCNAGSCIDLAYKYKLKSKKWKEVKNPFHVVCDVSOFSG ELHRFLVQPLNDGVKLIAVMDCCNAGSCIDLAYKYKLKSKKWKEVKNPFHVVCDVSQFSG ELHRFLVQPLNDGVKLIAVMDCCNAGSCIDLAYKYKLKSKKWKEVKNPFH』CDVSQFSG

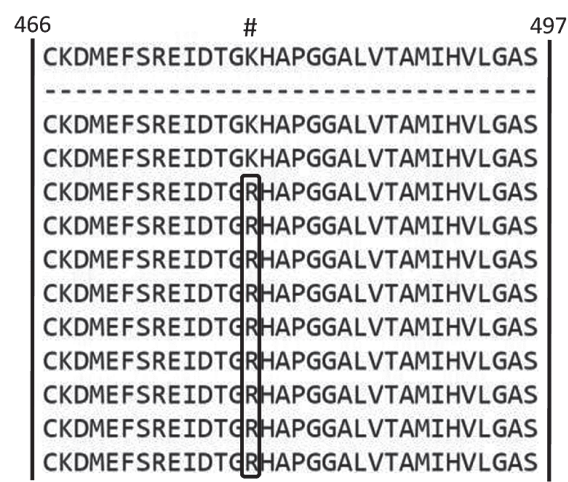

Fig. 1: multiple alignment of PvMCA1 peptidase domain from Brazilian Amazon field isolates and Plasmodium vivax strains from different endemic countries around the world. Deduced amino acid sequences of Peptidase_C14 domain of $P v$ MCA1 were obtained from $83 P$. vivax isolates collected in two municipalities of the Juruá Valley (CZS: Cruzeiro do Sul and; Mâncio Lima: ML) and were, then, compared against sequences deduced from 112 P. vivax nucleotide genomic sequences available in GenBank and PlasmoDB, using Sal-1 as reference strain. Selected sequences are shown and the complete analysis is available in Supplementary data. The canonical His372-Cys428 catalytic dyad and the substitutions of amino acid residues are indicated by (*) and (\#), respectively. $(\downarrow)$ indicates the adjacent cysteine residue (Cys427) with possible involvement in protease activity. CZS/ML represents all field isolates studied, since complete identity was observed. Monkey-adapted strains are represented by Sal1, BrazilI, MauritaniaI and IndiaVII; clinical isolates by Brazil32, PvC01, PvT01 and PvP01 and; wild ape isolates by PvSY42, PvSY56, Pv104 and Pvl10. (-): indicates non-determined amino acid residues. 

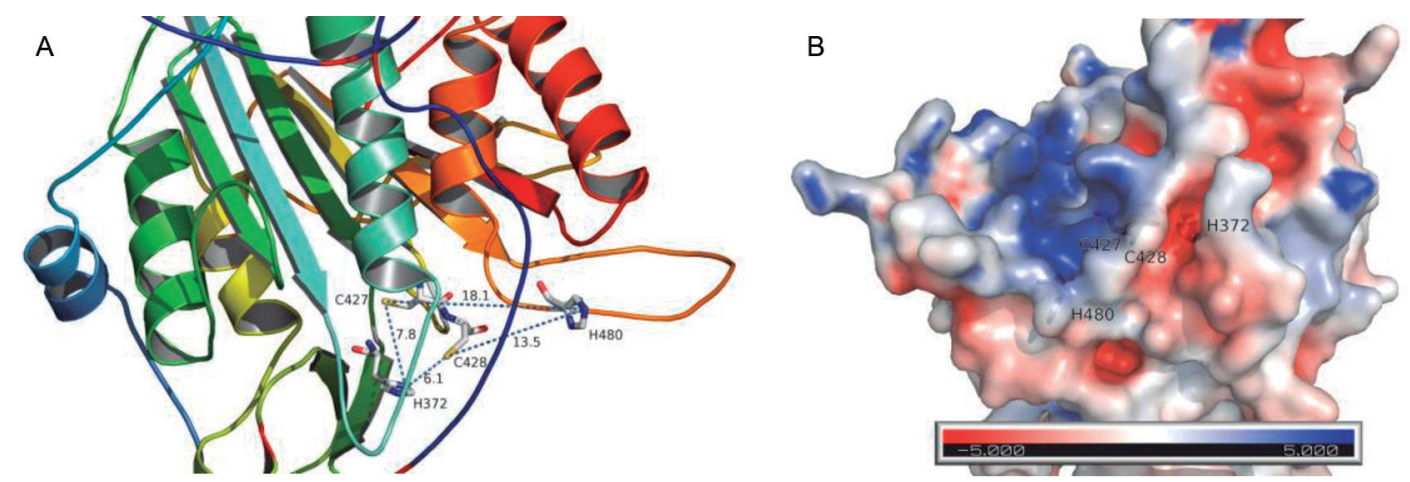

Fig. 2: prediction of catalytic dyad of $P v \mathrm{MCA} 1$ by molecular modeling. (A) Three-dimensional structure of $P v \mathrm{MCA} 1$ catalytic domain was modeled using comparative modeling and the distances (in $\AA$ ) between the putative amino acid residues participating in catalytic dyad formation were estimated considering the $\mathrm{N} \varepsilon$ atom of histidine $(\mathrm{H})$ imidazole ring and $\mathrm{S} \gamma$ atom of cysteine $(\mathrm{C})$, which are crucial for the proteolytic process. (B) Electrostatic surface potential analysis of $P v \mathrm{MCA} 1$ catalytic domain: negatively charged regions are shown in red and positively regions in blue.

laria in Manaus city, Amazonas state, does not indicate a relation between $P v \mathrm{MCA} 1$ genetic diversity and parasite chemoresistance, as no mutation in the protease catalytic domain was detected in these patients (data not shown). Even so, the conclusion of the survey with a larger number of samples appropriately characterised in terms of parasite drug-sensitivity is needed to better clarify this issue.

In order to know the variability of $P v \mathrm{MCA} 1$ worldwide, we extended our analysis to $P$. vivax isolates from different geographical regions whose genomic sequences are publicly available and, additionally, evaluated complete peptidase C14 domain (K286-S497), as predicted using Pfam database of protein families. Nucleotide sequence analysis of more than one hundred isolates from Americas, Africa, Asia and Indonesia, which included clinical and monkey-adapted strains as well as ape-infecting strains from Cameroon and Gabon (Supplementary data), revealed only four non-synonymous polymorphisms at position 310 (Leu to Val), 360 (Gln to Glu), 457 (Val to Met) and 479 (Lys to Arg) (Fig. 1). Three of them (L310V, Q360E, V457M) were restricted to wild ape parasites, with L310V substitution occurring markedly in all eight isolates analysed, while K479R substitution was the unique variation observed in the clinical and monkey-adapted isolates (Fig. 1 and Supplementary data). It is noteworthy that substitutions were not related to cysteine and histidine residues; even in the ape-infecting $P$. vivax strains, whose remarkable genetic diversity comparing to human strains suggests distinct demographic histories. ${ }^{(23)}$ These data indicate, therefore, that the peptidase domain of $P v \mathrm{MCA} 1$ presents evolutionary conservation of amino acid residues that are putatively involved in metacaspase activity, as described across diverse taxa. ${ }^{(24,25)}$

Similar results showing few polymorphic nucleotides with high conservation of essential amino acid residues required to catalytic site formation have previously been reported for other $P$. vivax proteases, such as vivapain-1, -2 and -3 , plasmepsin- 4 and $P v$ SERA- 4 . $(26,27,28)$ Curiously, the polymorphisms reported by Sow et al. ${ }^{(15)}$ in $P v$ MCAl peptidase domain were restricted to histidine and cysteine residues, including the putative H372-C428 catalytic dyad, which could impact protease activity. Abrogation of proteolytic activity can indeed be achieved by site-specific mutagenesis of histidine and cysteine residues forming consensus catalytic dyad in metacaspases of T. brucei (TbMCA2), Leishmania major (LmjMCA) and Candida albicans (CaMca1). ${ }^{(29,30,31)}$ But in other metacaspases this dyad seems not to be essential for catalytic activity, such as in $L d$ MCA1 of $L$. donovani ${ }^{(32)}$ and a secondary catalytic cysteine has been evidenced in T. congolense (TcoMCA5) as well as in the plant species Arabidopsis thaliana (AtMC9) and Triticum aestivum (TaeMCAII). ${ }^{(33,34,35)}$

This raises the possibility of alternative amino acid residues in $P v$ MCA1 compensating for the mutations previously reported by Sow and colleagues. ${ }^{(15)}$ For instance, in MCA1 of P. berghei, P. chabaudi and P. yoelii the predicted catalytic cysteine occurs immediately before of the consensus position, where a substitute residue (proline) is present. ${ }^{(14)}$ The same is true for T. brucei MCA4 (TbMCA4), in which the activity of such cysteine was experimentally demonstrated. ${ }^{(36)}$ Interestingly, $P v$ MCA1 (Fig. 1; Cys427) and other metacaspases, including MCA1 of $P$. knowlesi and $P$. gallinaceum, present a second conserved cysteine adjacently preceding the consensus catalytic cysteine ${ }^{(11,14)}$ and the involvement in proteolytic activity has been shown as well. ${ }^{(29,30,35)}$ Notably, substitution of this alternative cysteine is not found in any of the $P$. vivax isolates analysed until now (Cys427), indicating by its high degree of conservation a possible role in $P v$ MCA1 activity.

We then predicted the three-dimensional structure of $P v$ MCA1 catalytic domain by homology modeling to examine if $\mathrm{C} 427$ and additional histidine residues could, in some extent, participate in catalytic site formation. As shown in Fig. 2, the area harboring the canonical catalytic dyad (His372-Cys428) presented typical electrostatic potential of metacaspase catalytic pocket (Fig. $2 \mathrm{~B}$ ), which is endowed with negative charges and confers specificity toward substrates containing basic arginine or lysine residues ${ }^{(13,24)}$ while Cys427 was located in a more positively charged area (Fig. 2B). On the other hand, the proximity of both Cys427 (7.8 $\AA$ ) and Cys428 (6.1 $\AA$ ) to His372 residue was shown to be consistent with the active site reported for some cysteine proteases 
(Fig. 2A), such as legumains, caspases and metacaspases. ${ }^{(34,37,38)}$ In the single metacaspase of $S$. cerevisiae (YCA1), for instance, the distance between catalytic residues (Cys-His) was about $9.2 \AA$, while the nearest histidine $(5.8 \AA)$ was not implicated in the proteolytic process, ${ }^{(35)}$ supporting that Cys427 could participate in the activity of $P v \mathrm{MCA} 1$. In this context, an additional histidine residue (His480) was also identified in the vicinity of the canonical catalytic dyad but, unless a conformational change occurs before catalytic site activity, His 480 does not seem to constitute a partner residue for Cys 427 or Cys 428 , since it is $>13.0 \AA$ away (Fig. 2A).

In summary, although mutations in the putative catalytic dyad of $P v \mathrm{MCA} 1$ have previously been reported in field isolates from Mauritania, Sudan and Oman, a highly conserved peptidase domain of $P v \mathrm{MCA} 1$ was remarkably observed in Brazilian Amazon as well as between $P$. vivax isolates from different endemic countries around the world, which can contribute to eligibility of this metacaspase as a druggable target candidate in vivax malaria. Nevertheless, further studies aiming biochemical and functional characterisation of $P v \mathrm{MCA} 1$, together to identification of amino acid residues that effectively participate in the proteolytic process are still required to elucidate the role of $P v \mathrm{MCA} 1$ in $P$. vivax biology.

\section{ACKNOWLEDGEMENTS}

To DNA Sequencing facility of Fiocruz-RJ (P01-001) for the support in sequencing assays.

\section{AUTHORS' CONTRIBUTION}

Conceptualisation - HASS and PRRT; investigation and methodology - HASS, VFE, CMB, BOB, JPB and EKPR; formal analysis - ABMR, ACRG, FFM and JHMS; resources - GCM, MVGL, RMS, JCLJ, CTDR, LRPR and PRRT; writing - original draft preparation - HAS and PRRT; writing - review and editing - MVGL, CTDR, LRPR and PRRT; supervision - PRRT.

\section{REFERENCES}

1. Antony HA, Parija SC. Antimalarial drug resistance: an overview. Trop Parasitol. 2016; 6(1): 30-41.

2. WHO - World Health Organization. World Malaria Report 2019. Geneva: WHO; 2019. Available from: https://www.who.int/ publications/i/item/9789241565721.

3. Dondorp AM, Smithuis FM, Woodrow C, von Seidlein L. How to contain artemisinin- and multidrug-resistant falciparum malaria. Trends Parasitol. 2017; 33(5): 353-63.

4. Renapurkar DM, Pradhan VR, Sutar NK, Deshmukh RA, Pandit $\mathrm{CH}$, Marathe SN. Micro test for assaying sensitivity of Plasmodium vivax in vitro. Chemotherapy. 1989; 35(3): 160-3.

5. Rieckmann KH, Davis DR, Hutton DC. Plasmodium vivax resistance to chloroquine? Lancet. 1989; 2(8673): 1183-4.

6. Price RN, von Seidlein L, Valecha N, Nosten F, Baird JK, White NJ. Global extent of chloroquine-resistant Plasmodium vivax: a systematic review and meta-analysis. Lancet Infect Dis. 2014; 14(10): 982-91.

7. Dayananda KK, Achur RN, Gowda DC. Epidemiology, drug resistance, and pathophysiology of Plasmodium vivax malaria. J Vector Borne Dis. 2018; 55(1): 1-8.
8. Thomas D, Tazerouni H, Sundararaj KGS, Cooper JC. Therapeutic failure of primaquine and need for new medicines in radical cure of Plasmodium vivax. Acta Trop. 2016; 160: 35-8.

9. Levy MR, Chou SC. Inhibition of macromolecular synthesis in the malarial parasites by inhibitors of proteolytic enzymes. Experientia. 1975 ; 31(1): 52-4.

10. Rosenthal PJ. Proteases of malaria parasites: new targets for chemotherapy. Emerg Infect Dis. 1998; 4(1): 49-57.

11. Kumar B, Mohammad T, Amaduddin, Hussain A, Islam A, Ahmad F, et al. Targeting metacaspase-3 from Plasmodium falciparum towards antimalarial therapy: a combined approach of in-silico and in-vitro investigation. J Biomol Struct Dyn. 2021; 39(2): 421-30.

12. Vandana, Shankar S, Prasad KM, Kashif M, Kalia I, Rai R, et al. A nonpeptidyl molecule modulates apoptosis-like cell death by inhibiting $P$. falciparum metacaspase-2. Biochem J. 2020; 477(7): 1323-44.

13. Klemenčič M, Funk C. Structural and functional diversity of caspase homologues in non-metazoan organisms. Protoplasma. 2018; 255(1): 387-97.

14. Le Chat L, Sinden RE, Dessens JT. The role of metacaspase 1 in Plasmodium berghei development and apoptosis. Mol Biochem Parasitol. 2007; 153(1): 41-7.

15. Sow F, Bonnot G, Ahmed BR, Diagana SM, Kebe H, Koita M, et al. Genetic diversity of Plasmodium vivax metacaspase 1 and Plasmodium vivax multi-drug resistance 1 genes of field isolates from Mauritania, Sudan and Oman. Malar J. 2017; 16(1): 61.

16. Ferreira MU, Castro MC. Challenges for malaria elimination in Brazil. Malar J. 2016; 15(1): 284

17. Snounou G, Viriyakosol S, Zhu XP, Jarra W, Pinheiro L, Rosario VE, et al. High sensitivity of detection of human malaria parasites by the use of nested polymerase chain reaction. Mol Biochem Parasitol. 1993; 61(2): 315-20.

18. Biswas C, Zuo X, Chen SCA, Schibeci SD, Forwood JK, Jolliffe $\mathrm{KA}$, et al. Functional disruption of yeast metacaspase, Mcal, leads to miltefosine resistance and inability to mediate miltefosine-induced apoptotic effects. Fungal Genet Biol. 2014; 67: 71-81.

19. de Oliveira TC, Corder RM, Early A, Rodrigues PT, Ladeia-Andrade S, Alves JMP, et al. Population genomics reveals the expansion of highly inbred Plasmodium vivax lineages in the main malaria hotspot of Brazil. PLoS Negl Trop Dis. 2020; 14(10): e0008808.

20. Tsiatsiani L, Van Breusegem F, Gallois P, Zavialov A, Lam E, Bozhkov PV. Metacaspases. Cell Death Differ. 2011; 18(8): 1279-88.

21. Picot S, Burnod J, Bracchi V, Chumpitazi BF, Ambroise-Thomas P. Apoptosis related to chloroquine sensitivity of the human malaria parasite Plasmodium falciparum. Trans R Soc Trop Med Hyg. 1997; 91(5): 590-1.

22. Meslin B, Beavogui AH, Fasel N, Picot S. Plasmodium falciparum metacaspase PfMCA-1 triggers a z-VAD-fmk inhibitable protease to promote cell death. PLoS One. 2011; 6(8): e23867.

23. Loy DE, Plenderleith LJ, Sundararaman SA, Liu W, Gruszczyk J, Chen YJ, et al. Evolutionary history of human Plasmodium vivax revealed by genome-wide analyses of related ape parasites. Proc Natl Acad Sci USA. 2018; 115(36): E8450-9.

24. Klemenčič M, Asplund-Samuelsson J, Dolinar M, Funk C. Phylogenetic distribution and diversity of bacterial pseudo-orthocaspases underline their putative role in photosynthesis. Front Plant Sci. 2019; 10: 293.

25. Kumar B, Verma S, Kashif M, Sharma R, Atul, Dixit R, et al. Metacaspase-3 of Plasmodium falciparum: an atypical trypsinlike serine protease. Int J Biol Macromol. 2019; 138: 309-20. 
26. Na BK, Shenai BR, Sijwali PS, Choe Y, Pandey KC, Singh A, et al. Identification and biochemical characterization of vivapains, cysteine proteases of the malaria parasite Plasmodium vivax. Biochem J. 2004; 378(Pt 2): 529-38.

27. Rawat M, Vijay S, Gupta Y, Dixit R, Tiwari PK, Sharma A. Sequence homology and structural analysis of plasmepsin 4 isolated from Indian Plasmodium vivax isolates. Infect Genet Evol. 2011; 11(5): 924-33.

28. Rahul CN, Shiva Krishna K, Pawar AP, Bai M, Kumar V, Phadke $\mathrm{S}$, et al. Genetic and structural characterization of PvSERA4: potential implication as therapeutic target for Plasmodium vivax malaria. J Biomol Struct Dyn. 2014; 32(4): 580-90.

29. González IJ, Desponds C, Schaff C, Mottram JC, Fasel N. Leishmania major metacaspase can replace yeast metacaspase in programmed cell death and has arginine-specific cysteine peptidase activity. Int J Parasitol. 2007; 37(2): 161-72.

30. Moss CX, Westrop GD, Juliano L, Coombs GH, Mottram JC. Metacaspase 2 of Trypanosoma brucei is a calcium-dependent cysteine peptidase active without processing. FEBS Lett. 2007; 581(29): 5635-9.

31. Jeong JH, Lee SE, Kim J. Mutational analysis of metacaspase CaMcal and decapping activator Edc3 in the pathogenicity of Candida albicans. Fungal Genet Biol. 2016; 97: 18-23.

32. Lee N, Gannavaram S, Selvapandiyan A, Debrabant A. Char- acterization of metacaspases with trypsin-like activity and their putative role in programmed cell death in the protozoan parasite Leishmania. Eukaryot Cell. 2007; 6(10): 1745-57.

33. Belenghi B, Romero-Puertas MC, Vercammen D, Brackenier A, Inzé D, Delledonne M, et al. Metacaspase activity of Arabidopsis thaliana is regulated by S-nitrosylation of a critical cysteine residue. J Biol Chem. 2007; 282(2): 1352-8.

34. Piszczek E, Dudkiewicz M, Mielecki M. Biochemical and bioinformatic characterization of type II metacaspase protein (TaeMCAII) from wheat. Plant Mol Biol Report. 2012; 30(6): 1338-47.

35. Eyssen LEA, Coetzer THT. Expression, purification and characterisation of Trypanosoma congolense metacaspase 5 (TcoMCA5) - a potential drug target for animal African trypanosomiasis. Protein Expr Purif. 2019; 164: 105465.

36. Szallies A, Kubata BK, Duszenko M. A metacaspase of Trypanosoma brucei causes loss of respiration competence and clonal death in the yeast Saccharomyces cerevisiae. FEBS Lett. 2002; 517(1-3): 144-50.

37. Wong AHH, Yan C, Shi Y. Crystal structure of the yeast metacaspase Yca1. J Biol Chem. 2012; 287(35): 29251-9.

38. Elsässer B, Zauner FB, Messner J, Soh WT, Dall E, Brandstette $\mathrm{H}$. Distinct roles of catalytic cysteine and histidine in the protease and ligase mechanisms of human legumain as revealed by DFTbased QM/MM simulations. ACS Catal. 2017; 7(9): 5585-93. 